\title{
Les écritures migrantes au Québec: I'entre-deux selon Régine Robin
}

\author{
As escrituras migrantes no Québec: o entre-lugar segundo Régine Robin
}

\author{
Adina Balint-Babos
}

Université de Winnipeg - Canadá

$\odot$

\begin{abstract}
Résumé: Dans la mouvance d'une esthétique postmoderne, les littératures contemporaines du Canada, trop longtemps sourdes aux minorités qui les peuplent, s'ouvrent dans les années 1980 à la prise de parole d'écrivains nés ailleurs, qui choisissent le français ou l'anglais comme langue d'écriture. Au Québec, on parle d"“écritures migrantes", dont la diversité et la richesse sont notables. Ainsi, nous analysons le cas de Régine Robin, écrivaine qui n'est pas née au Québec, et qui réfléchit à l'entre-deux cultures et à l'identité plurielle, qui sont le lot des nouveaux arrivants dans les Amériques. La Québécoite (1983) présage en quelque sorte bien des idées sur le multiculturalisme et le transculturalisme.
\end{abstract}

Mots-clés: Entre-deux; Écritures migrantes; Québec; Amériques

Resumo: Influenciadas por uma estética pós-moderna, as literaturas contemporâneas do Canadá, que por muito tempo ignoraram as minorias que as povoam, abrem-se, nos anos 1980, ao discurso de escritores nascidos no exterior que escolheram o francês ou o inglês como língua de escrita. No Quebec, fala-se de "escritas migrantes", cuja diversidade e riqueza são notáveis. Assim, analisamos o caso de Régine Robin, escritora não nascida no Quebec que reflete sobre o entremeio cultural e a identidade plural, destino dos recém-chegados às Américas. La Québécoite (1983) adianta, de certa maneira, muitas ideias sobre o multiculturalismo e o transculturalismo.

Palavras-chave: Entremeio; Escritas migrantes; Quebec; Américas

La parole immigrante inquiète. Elle ne sait pas poser sa voix. Trop aiguë, elle tinte étrangement. Trop grave, elle déraille. Elle dérape, elle s'égare, s'affole, s'étiole, se reprend sa pudeur [...]. La parole immigrante dérange. Elle déplace, transforme, travaille, travaille le tissu même de cette ville éclatée. Elle n'a pas de lieu. Elle ne peut que désigner l'exil. L'ailleurs, le dehors [...]. La parole immigrante est insituable, intenable. Elle n'est jamais là où on la cherche, où on la croit. Elle ne s'installe pas. Parole sans territoire et sans attache [...]. On ne peut pas l'accrocher.

(ROBIN, 1983, p. 43)

\section{Introduction}

Nous autres, vous autres, eux autres... Parler de ce qu'on est, d'où on vient, du groupe auquel on appartient au Québec passe par l'utilisation du mot “autre". Il s'agit d'inscrire constamment une différence en employant le pluriel: "nous", "vous", "eux", jamais "moi autre". La formule "nous autres" marque ainsi une altérité entretenue, choisie, entrée dans l'usage; c'est dire l'appartenance à un groupe défini autour d'un "nous" identitaire, alors que "vous autres", "eux autres" sont ceux qui ne sont pas comme "nous". On voit surgir ici plusieurs questions qu'on propose d'examiner à travers le roman La Québécoite de Régine Robin: la question de l'identité et de l'altérité (celui qui est né ici et celui qui vient d'ailleurs), l'inclusion-exclusion, le groupe dominant et les autres... Quelle que soit l'interrogation autour du dualisme, elle demande à être nuancée. Pourquoi? Parce qu'à l'heure du monde globalisé, il est désormais connu que la littérature contemporaine au Québec ne privilégie plus la souche, les discours identitaires fermés sur euxmêmes; cette littérature examine des perspectives multi 
et transculturelles, elle "se déplace dans le contexte de la légitimation des déplacements géographiques et symboliques. [...] elle tend à explorer les facettes des diversités planétaires"1. Ou encore, comme le souligne Patrick Imbert, dans cette littérature, "on cherche à comprendre les valeurs différentes et voir comment peut s'établir un vivre-ensemble efficace émotionnellement, économiquement et intellectuellement" ${ }^{2}$. On comprend ainsi que l'important est de saisir des passerelles, des liens avec les autres, d'entamer des rencontres, plutôt que de mettre de l'avant un discours d'identité-singularité qui exclut dès qu'on prononce "nous autres" vs. "vous autres", "eux autres". Dans cette perspective, le titre de l'essai récent de Régine Robin Nous autres, les autres, paru en 2013, montre justement qu'il est possible de disloquer, déplacer, détourner le dualisme; "nous autres, les autres" subvertit l'idée de nationalisme québécois, et nous conduit à changer l'angle de vue, à poser le regard sur le groupe des autres, pas d'emblée sur le groupe dominant $^{3}$.

Si le terme "écriture migrante" va de soi aujourd'hui - quand il s'agit de parler d'écrivains qui vivent et qui écrivent au Québec, mais qui sont nés ailleurs -, dans les années 1980, cette expression ne s'est pas imposée immédiatement. Il y a eu des tâtonnements dans la recherche d'une définition, ce qui dit bien l'espace incertain de la jeune culture québécoise (SIMON et al., 1991, p. 26); on a parlé d'abord d'écriture ethnique, d'écriture métisse ou métissée, immigrée et/ou immigrante, hybride ou bien, plus banalement, de littérature néo-québécoise. En réalité, c'est un calque de l'anglais migrant writing qui désigne une forme littéraire en acte, in progress, de la part d'écrivains qui migrent d'un lieu à l'autre, entre un lieu de départ et un lieu d'arrivée (SIMON et al., 1991, p. 57). De cette situation d'entre-deux naît chez l'individu (et chez l'écrivain.e) un sentiment de désorientation physique et psychique, d'étrangeté par rapport au lieu d'élection, et par conséquent, de déterritorialisation.

Pourquoi donc cette forme littéraire prend-elle corps au Québec dans les années 1980, au lendemain d'un référendum pour l'Indépendance qui a échoué? Une génération d'intellectuels québécois avait pourtant participé activement à la Révolution Tranquille, vingt ans plus tôt, et à l'élaboration d'un texte national homogène, dotant ainsi le pays d'une littérature aux racines québécoises, se séparant du patrimoine littéraire

\footnotetext{
1 Patrick Imbert, Imprévus et coüncidences. Le Canada et les Amériques, Ottawa, Editions de la Chaire de recherche de l'Université d'Ottawa: Canada: enjeux sociaux et culturels dans une société du savoir, 2013 , p. 9.

2 Ibid., p. 10

3 Voir aussi Chez Elena Botchorichvili dans Seulement attendre et regarder, cette inquiétude dans le clivage dualiste: "Eux? Nous? Nous, c'est nous, vous c'est vous? Vous c'est vous, nous c'est nous? “ (p. 47).
}

français qui avait dominé jusqu'alors. Par ailleurs, dans les années 1960, la plupart des écrits (auto)biographiques s'enracinent dans la "terre de Québec" et mythifient la ville de Montréal. Ce discours identitaire, teinté de nationalisme, s'essouffle assez vite, les écrivains tendent à se replier sur eux-mêmes, après l'échec du référendum, et c'est alors que fait brèche une expression de l'hétérogène (L'HĖRAULT, 1991, p. 57), de la part d'écrivains qui ne sont pas de souche. Les premiers sont les Italiens, les plus nombreux comme communauté, qui avancent sur la scène culturelle montréalaise et fondent en 1983 une revue transculturelle qui porte un titre latin, Vice Versa, animée par des intellectuels nés en Italie: Antonio d'Alfonso, Marco Micone, Lamberto Tassinari, Fulvio Caccia et son frère Gianni Caccia, d'autres encore, et Régine Robin, Française née à Paris de parents juifs polonais, arrivée au Québec quelques années auparavant - tous émigrés ou fils d'émigrés, en mesure, par leur culture, de prendre conscience de leur condition. Vice Versa - revue ouverte à l'Autre, graphiquement très créative, écrite en trois langues - l'italien, le français et l'anglais, lance un message original, frayeur de chemins:

\begin{abstract}
Que cache-t-elle, cette émigration, pour qu'on puisse la voir comme une des causes de ce nouveau cosmopolitisme, et peut-être de ce nouvel humanisme, qui commence à prendre forme dans nos villes? Elle cache la fin de l'ethnicité. [...] l'émigrant a une manière nouvelle de se percevoir par rapport à ses origines, à sa terre, à sa langue, à sa présence ici $[. .$.$] sa perte$ du territoire d'origine est peut-être sa damnation [...] mais aussi sa grande force. Sans le savoir, sans même le soupçonner, les premiers émigrants ont amorcé le processus transculturel. Leur migration a agi comme une véritable bombe à retardement dont l'éclatement est l'émergence des nouvelles générations. (TASSINARI, 1986 et 2005)
\end{abstract}

Toujours dans les années 1980, une deuxième brèche importante au Québec s'impose sous l'influence des Women studies américaines: les femmes longtemps marginalisées, reléguées dans des revues féminines, genre La veillée des chaumières, commencent à écrire des récits de vie et apportent un appui décisif à la mythisation de Montréal en décrivant la ville selon un célèbre triangle "corps-ville-littérature" (BROSSARD et GIROUARD, 1992).

Le roman La Québécoite de Régine Robin paraît la même année que Vice Versa, mais il est en gestation depuis 1974, et s'adresse à un public québécois jeune, comme nous le dit la dédicace: "À mes étudiants des universités du Québec à Montréal, de Montréal, de Mc Gill, de Laval et de Sherbrook. 1974-1981". Il s'agit d'un regard nouveau sur Montréal, une écriture dérangeante 
qui brise les canons du français, en mettant en lumière le sentiment dysphorique de non-appartenance au lieu et à l'histoire du Québec, en cherchant une issue dans l'errance et la fuite discursives et thématiques.

La Québécoite connaît un succès considérable. Ce livre constitue aujourd'hui un incontournable dans l'analyse du phénomène de "l'écriture migrante" et des problèmes que pose la nouvelle posture de l'écrivain québécois depuis les années 1980. Or, il est intéressant de noter que dans La Québécoite, la narratrice utilise le mot "autre" pour faire part de sa solitude et de sa marginalité: "Quelle angoisse certains après-midis Québécité, québécitude - je suis autre. Je n'appartiens à ce Nous si fréquemment utilisé ici - Nous autres Vous autres" (ROBIN, 1983, p. 53-54). Exclue du Nous englobant, la narratrice est également exclue du Vous tout aussi englobant. Rejetée d'une identité communautaire qu'elle ne peut et ne veut pas assimiler, elle erre dans son espace à elle qui n'est ni ici ni là-bas. C'est un territoire dont les frontières ont éclaté sous le choc de l'exil et qui est désormais impossible à délimiter. La narratrice et le personnage du récit cherchent en vain ce qui a été perdu: l'identité d'avant, un sentiment d'appartenance, quelque chose qui serait possible à cerner et qui mettrait fin à l'errance.

Ainsi, l'espace de La Québécoite ne se forge pas directement au croisement des deux identités, mais surgit de la recherche, de l'oscillation constante entre ces deux identités. Ce territoire engendré par le mouvement de va-et-vient est ce qu'on désignera, d'après les mots de Robin, par "l'entre-deux". C'est par cet entre-deux en quête d'un sentiment d'appartenance que se raconte l'expérience migrante. Des critiques ont souligné la prégnance de l'entre-deux dans le roman, sans pour autant penser l'éclatement des espaces topique, langagier et mémoriel pour rendre compte de l'étrangeté de la solitude qui est le lot de l'exilée. Et c'est justement de juive errante qu'on pourrait qualifier la Québécoite qui, en se déplaçant constamment, crée un territoire qui lui est propre, dans les plis de l'entre-deux; dans l'espoir d'appartenir. Par ailleurs, Patrick Imbert, dans Comparer le Canada et les Amériques, souligne avec justesse l'idée d'inachèvement - donc des allées et venues, des tâtonnements - quand il s'agit de penser les identités et les récits issus des Amériques:

[...] les Amériques constituent encore un projet inachevé. Elles produisent constamment des récits neufs qui prennent en charge les mobilités culturelles et les multiples rencontres dans des dynamiques ouvertes à l'imprévu. Ces récits neufs sont liés aux perspectives que proposent l'interculturalisme, le multiculturalisme et le transculturalisme. (IMBERT, 2014, p. 4)
Pourtant, que signifie “appartenir"? C'est une interrogation qui revient sous une forme ou une autre à travers toute l'œuvre philosophique de Jacques Derrida. On y entend aussi bien la volonté d'interroger les crispations identitaires actuelles que la remise en cause de toute idée d'appartenance, qu'elle soit nationale, linguistique, communautaire ou sexuelle. Chez Derrida, la question est au moins double: premièrement, que restet-il de nos appartenances, de l'héritage commun, de ce qui, d'une certaine façon et selon des voies multiples, nous fait "tenir ensemble"? Question nostalgique mais qui, plus largement, recouvre celle du sens partagé de la communauté. Deuxièmement: comment réinventer des nouvelles formes d'appartenance, sous quelles conditions, dans quelles limites? Dans la perspective de notre propos, nous pourrions ajouter: "dans les Amériques". Question qui concerne l'à venir, comme disait Derrida, et là encore, plus largement le sens à donner à toute œuvre - littéraire ou philosophique - œuvre d'art ou œuvre de vie. D'une part, le refus de l'appartenance au sens de l'identité, de l'enracinement, du propre, du soi-même comme un; et d'autre part, la nécessité de se forger une appartenance par la créativité et la création artistique et de pensée, une dynamique qui se manifeste déjà chez Oswald de Andrade au Brésil notamment dans son Manifeste anthropophage quoiqu'il y ajoute une perspective de dévoration/ recombinaison à visées transculturelles. Dans cet entredeux, on reconnaît également les questionnements de Régine Robin dans la Québécoite, mais aussi au fil de son œuvre. Chez Robin et chez Derrida, l'appartenance tient conjointement de l'impossible et du nécessaire. La question n'est pas simple, elle est autant théorique qu'intimement douloureuse. On se souvient de la belle formule de Circonfession: "qui suis-je si je ne suis pas ce que j'habite et où j'ai lieu?"4.

\section{L'espace topique: Montréal, ville éclatée}

Venons-en à ce curieux roman La Québécoite qui n'est pas un roman mais plutôt "une expérimentation à la fois littéraire et sociale" dans le but de "fictionnaliser l'inquiétante étrangeté que crée le choc culturel" (ROBIN, 1993, p. 207). C'est le récit d'une arrivée en ville, à Montréal, épisode-clef de tout récit d'immigration. Montréal, ville cosmopolite, ville longtemps muette et figée, vouée au commerce, ville divisée entre deux religions, deux langues nationales, deux systèmes scolaires confessionnels (le français, école catholique), l'anglais (école protestante et catholique irlandaise). La ville est partagée en deux par le Boulevard St. Laurent, frontière urbaine, linguistique,

\footnotetext{
4 "Circonfession", dans Jacques Derrida et Geoffrey Bennington (éds.) Jacques Derrida, p. 32.
} 
anagraphique; à l'est habitent les francophones, à l'ouest les anglophones, aujourd'hui la scission est beaucoup moins drastique. Ce boulevard sera le lieu d'ancrage des immigrés dans l'attente de rejoindre leurs communautés, on le nommait autrefois "Boulevard de la colonie", "Boulevard des émigrants", "Couloir de la colonie" ou encore La Main, en anglais.

Toutes ces précisions sont nécessaires pour comprendre le parcours que va accomplir la narratrice - jeune professeure française d'origine juive qui arrive de Paris pour enseigner dans une université d'abord anglophone puis francophone. Étrangère à cet espace à la fois double et multiple par toutes les langues qui s'y parlent, elle s'engage à fixer cette étrangeté avant qu'elle ne lui devienne familière:

Il fallait fixer tous les signes de la différence ; la différence des odeurs, de la couleur du ciel, la différence de paysage. Il fallait faire un inventaire, un catalogue, une nomenclature. Tout consigner pour donner plus de corps à cette existence. (ROBIN, 1983, p. 18)

Elle ne va pas cesser de traverser Montréal en métro ou en bus - la traversée de la ville d'ouest en est avec le bus no. 24 est très connue - en énumérant systématiquement les stations de métro, le nom des rues, les grands magasins, les affiches, la liste des partis politiques canadiens, les banques, les catéchismes, les émissions de télévision, toutes choses qui, à la fin du compte, ne lui disent rien. La vision qu'elle donne de Montréal est celle d'une ville tristement morcelée et juxtaposée. Voici la disposition graphique qu'elle en donne, en majuscules:

\section{DES GHETTOS \\ CHACUN SA LANGUE SA COMMUNAUTÉ \\ CHACUN SON QUARTIER SON DEPUTÉ \\ SES GATEAUX. SON JOURNAL. SA RELIGION SON FOLKLORE, SES POMPONS. CHACUN SON HISTOIRE SEULS \\ À PART \\ NOUS. VOUS. EUX. (ROBIN, 1983, p. 152-153)}

Vision dysphorique et fragmentée - cette citation en est la preuve patente - de mondes, de langues qui se succèdent au fil du paysage montréalais et où l'immigrée n'a pas sa place. Cette ville ne lui appartient pas, elle ne s'y reconnaît pas. La corporéité de son regard n'est pas en harmonie avec son corps et son moi. Ici commence l'errance.

La Québécoite est un récit sur l'exil qui reprend sans cesse le motif du déplacement afin d'évoquer l'éclatement identitaire inhérent à l'expérience de l'immigrante. La fragmentation identitaire ne se termine jamais et engendre dans son perpétuel recommencement la fragmentation de la ville. Dans ce récit, Montréal devient plurielle, toujours une autre. C'est d'ailleurs en vain que la narratrice cherche à habiter la ville qui lui échappe constamment, qui se démembre dès qu'elle cherche à se l'approprier. Or, pour l'exilée mise en scène dans La Québécoite, être ici, c'est aussi être là-bas. Les deux espaces se superposent, les couches spatiales en viennent à former des strates entre lesquelles le personnage s'égare, en errance entre différents quartiers de Montréal, entre Montréal et Paris, jamais vraiment ici, encore un peu là-bas.

De fait, la structure tripartite du récit participe de cet écartement spatial. Chacun des trois chapitres, dont les titres ("Snowdon", "Outremont", "Autour du marché Jean-Talon") évoquent des quartiers montréalais fort différents, présente le personnage pris dans une existence qui lui reste en partie étrangère. Ne pas cadrer, ne pas être encadrée, ne pas habiter d'espace clos semble être le lot de l'exilée étrangère ici, étrangère là-bas, étrangère parce qu'elle passe sans cesse d'un lieu à un autre: "Devenir étranger, c'est se percevoir en rupture de ban, se situer somme toute à l'écart d'un lieu circonscrit", souligne Simon Harel.

Mais pourquoi donc choisir le territoire mouvant de l'entre-deux, de l'oscillation identitaire? En réinventant constamment la vie, mais surtout l'espace de son personnage, la narratrice de La Québécoite semble chercher à échapper à la fixité identitaire signifiée par l'immobilisation du corps: "Ton personnage doit bien avoir quelques contradictions [...] il doit bien y avoir du manque quelque part. Ses déambulations même dans la ville insinuent le clivage" (ROBIN, 1983, p.60). Errant dans la ville, le personnage est à la recherche d'un lieu qui lui corresponde, qui le définisse; il est pris dans une quête impossible.

Par ailleurs, comment occuper cette ville à jamais insaisissable? En prouvant qu'elle existe bel et bien, qu'elle ne s'efface pas totalement dans son morcellement. Que les morceaux qui la constituent, si elles ne s'imbriquent pas les unes dans les autres, sont tout de même possibles à désigner. L'énumération se pose dès lors comme un moyen d'appréhender le monde étrange qui entoure la narratrice. "Seule l'accumulation de noms de rues, d'indices référentiels permet d'accréditer cette idée que la Montréal décrite est bien réelle. [...] Tout le roman de Régine Robin est traversé par cette obsession du lieu impossible à fixer"6. Et c'est par le biais de l'énumération

\footnotetext{
5 Simon Harel, "La parole orpheline de l'écrivain migrant", dans Pierre Nepveu et Gilles Marcotte (éds.), Montréal imaginaire. Ville et littérature, Montréal, Fides, 1992, p. 393.

6 Simon Harel, "Montréal: une 'parole' abandonnée. Gérard Étienne et Régine Robin”, p. 165.
} 
que s'inscrit l'accumulation dans La Québécoite, que la narratrice cherche à fixer l'espace urbain en mouvance constante. Montréal, qui se désintègre et se fragmente au contact de l'exilée, se présente à celle qui veut l'habiter comme un ensemble de signes possibles à désigner, à nommer:

Chez grand-mère - omelettes
Le Colbert: crêpes
À la crêpe bretonne
Le bistrot
La cabane à sucre
Le lancelot
Bar le cachet
Le fou du roi
La crêpe aux bleuets et gwennerch h $h$ crêpe à la pâte
d'amande avec crème Chantilly flambée au kirsch:
joyau de la maison - 5 dollars 75 cents.
(ROBIN, 1983, p. 55 )

Cette énumération des restaurants de la rue de la Montagne, si elle permet d'attester de la présence de la narratrice à cet endroit, achoppe pourtant. Ce que la narratrice cherche à raconter par l'entremise de son catalogage, c'est bien son désir de "lutter contre l'effacement des choses, tout en redonnant à cette ville sans temps ni lieu une certaine unité" 7 . Pourtant, même une fois réduite à un simple répertoire, la ville demeure opaque et inaccessible, ne rendant possible pour l'immigrante que l'errance.

On comprend ainsi que l'espace de l'exilée est un lieu qu'elle seule peut habiter pour demeurer ce qu'elle est, à savoir une figure de l'errance. Un espace s'ouvre donc malgré tout dans la ville parcourue. Le mouvement crée un territoire qui n'est ni ceci, ni cela, qui est entre ceci et cela et qui offre au personnage de La Québécoite un refuge instable mais un refuge tout de même:

Ce serait trop simple - à peine arrivée installée comme depuis toujours [...] - Ce pays - opaque - Tu le savais [...]. Tu auras été portée, happée, dévorée à la va-comme-je-te-pousse. Rejetée la plupart du temps, refusée - défaite, refaite - la porosité des lieux à t'envahir - sans ordre, ni chronologie, ni logique. (ROBIN, 1983, p. 52)

Le sujet est à l'image de la ville, en constante mutation, puisqu'il se voit envahi d'une "inquiétante étrangeté". Montréal incarne ainsi la traversée infinie qu'est l'exil. Le mouvement de l'immigrante rend possible l'engendrement d'un espace indéfini où l'autre est sans cesse en déplacement. Or, si le thème de l'entredeux prend une forme territoriale pour le personnage, il relève du langage pour la narratrice néanmoins, il reste à l'image de l'entre-deux topique, un espace qui se dérobe à la volonté de l'autre.

\section{L'espace langagier: "Aucun lieu/tout juste une voix plurielle" (ROBIN, 1983, p. 167)}

Dans ce mouvement constant entre ceci et cela, la prise de parole chez Robin cherche en vain à instaurer un équilibre. Si écrire c'est ouvrir dans le langage un espace à soi, le lieu engendré par la narratrice de La Québécoite éclate à l'image de la Montréal disloquée: "Serait-il possible de trouver une position dans le langage, un point d'appui, un repère, un point stable, quelque chose qui ancre la parole alors qu'il n'y a qu'un tremblé de texte, une voix muette, des mots tordus" (ROBIN, 1983, p. 18). Le langage apparaît comme un possible lieu de stabilité; or, la fragmentation identitaire ne peut se dire que par la fragmentation langagière.

Au pôle opposé, la langue-identité de la narratrice est celle de l'origine, de sa mère; celle d'avant la séparation et le désordre. C'est le yiddish: "Dans le fond, tu as toujours habité un langage et aucun autre ailleurs - ces petites taches noires sur le papier que l'on lit de droite à gauche. [...]. Ceux [ces signes] de ton enfance, de ta mère, de ton seul pays ce langage. Un langage à l'envers, allant vers on ne sait quoi" (ROBIN, 1983, p. 139-140). Il s'agit ici d'une langue qu'il est possible d'habiter, qui se laisse incorporer et dans le sein de laquelle la narratrice trouve un espace originel unifiant. Cette langue n'évoque pas l'éclatement et l'angoisse de l'étrangeté, mais bien une sorte de mouvance bienveillante, l'unité et le familier. Pourtant, l'ordre a été rompu, et par le fait même, l'identité fragmentée par l'exil. Il n'est pas possible de rendre compte de l'expérience de l'immigration avec ce langage d'avant le désordre. Poser l'acte d'écriture, c'est le moyen pour la narratrice non pas pour réinstaurer l'unité originelle, mais bien pour en montrer l'impossibilité. Le projet d'écriture est de fait voué à l'échec: "Elle aurait commencé un roman impossible" (ROBIN, 1983, p. 54).

En l'occurrence, à l'image de l'espace topique qui éclate au contact de l'autre, l'espace langagier vole en éclats lorsqu'il doit rendre compte de l'exil: "pour l'écrivain migrant, écrire serait accepter de mettre en crise le langage. Dans cette perspective, l'expérience de l'exil se traduira par une modification particulièrement nette des structures narratives qui prêtent forme à l'écrit", souligne encore Simon Harel. Ici, il n'y a pas de structure. La Québécoite s'ouvre d'ailleurs sur un acte de foi: l'incipit annonce le projet sous le signe duquel s'élaborera le récit: "Pas d'ordre" (ROBIN, 1983, p. 15). Dans le roman de Robin, le sujet écrivant est clivé, fracturé.

\footnotetext{
7 Madeleine Frédéric, "L'Écriture mutante dans La Québécoite de Régine Robin", dans Benoît Melançon et Pierre Popovic (éds.), Montréal 16421992. Le Grand passage, Montréal, XYZ, 1994, p. 496.

8 Simon Harel, "La parole orpheline de l'écrivain migrant", p. 385.
} 
La Québécoite est à la fois celle qui écrit et celle dont on raconte l'histoire; celle qui dit en même temps je et $t u$ et elle, errant parmi ces différentes identités: "Elle ne saurait jamais où la porteraient ses pas. [...] - écartèlement des cultures je suis à califourchon [...]. Toi perdue, à nouveau l'errance. Depuis toujours nous sommes des errants" (ROBIN, 1983, p. 63). Ce glissement du elle au je, puis au $t u$ se conclut par l'utilisation du nous, annonçant le lien étroit entre ces trois femmes, ou plutôt l'éclatement de la femme à la fois personnage, narratrice et interlocutrice.

Après tout, le récit en tant qu'espace pour dire l'errance, menace de se transformer à tout moment en demeure, en domicile, en logis douillet où la narratrice, fatiguée de ses oscillations constantes entre ceci et cela, a envie de trouver le repos: "Impossible à fixer dans cette géographie urbaine, dans cet espace mouvant. Dès qu'elle est installée, intégrée, elle s'enfuit, déménage et m'oblige à casser le récit alors que je commençais à m'y installer moi-même, à y prendre goût, à me reposer. Elle prend corps et dès lors s'enfuit, me fait la nique" (ROBIN, 1983, p. 138). Il est évident que les possibles accablent le récit et prennent à bras-le-corps la narratrice, suivant le personnage nomade dans son mouvement vers un ailleurs in-désignable. À cela s'ajoute la mémoire qui, à son tour, se joue de l'exactitude des remémorations qui, elles aussi, oscillent entre différents possibles.

\section{L'espace mémoriel: quand le temps rompt la chronologie}

La Québécoite reprend constamment un topos que l'on pourrait qualifier de superposition d'existences, une dynamique qui joue de l'accumulation par strates et des mélanges sans synthèse élégante comme le constatent de nombreux auteurs des Amériques, notamment Isabel Allende dans Hija de la fortuna lorsqu'elle explore l'accumulation des rencontres et leurs traces mémorielles dans la protagoniste voyageant du Chili à la Californie. Dans le récit, tout se superpose, s'ajoute à ce qui le précède. Ainsi, les espaces s'accumulent, les histoires s'additionnent les unes aux autres. Il en va de même pour la mémoire où s'enchevêtrent passé, présent et désir d'avenir dans une temporalité éclatée, qui rompt la chronologie. Chez Robin, l'existence se vit comme une continuité dans le désordre créatif. L'éclatement temporel est étroitement lié à l'éclatement mémoriel, puisque certains souvenirs en viennent à se greffer au présent morcelé et instable associé à l'immigration. Ces intermittences appellent à la fois l'éclatement spatial puisque le présent est vécu à Montréal, dans cette ville où surgissent les souvenirs vécus ailleurs: "Montréal semble permettre la sédimentation des mémoires, elle qui se caractérise par sa porosité alors que Paris tient lieu de centre métropolitain relativement immuable, elle est la ville de l'enfance et des années de formation. [...]. Ville centrale dans La Québécoite de Régine Robin, mais qui est toujours perçue à travers le prisme montréalais" rappelle Simon Harel.

Après tout, vivre dans une de ces villes nécessite le souvenir de l'autre ville, celle laissée derrière; les souvenirs se superposent comme les espaces auxquels ils sont associés, multipliant l'identité de l'immigrante. Paris et Montréal sont ainsi étroitement liées malgré la place excessivement différente que ces villes occupent dans la mémoire de la narratrice et du personnage qui glissent d'une ville à une autre, d'une histoire à une autre, d'une culture à une autre:

Il y avait les CANONS/le CANON de TOLBIAC/ et/les BOUQUET DE L'OPÉRA/LE BOUQUET DE VERSAILLES/LE BOUQUET DE L'OPÉRA (...)/sans compter LES CHIENS/ PENDANT LA GUERRE. Comme le ratage de l'exil même. [...] Un express bien tassé - bon - je me taille. Je me calte. Je lève l'ancre. Je fiche le camp. Je me tire. Je mets des bouts. Je mets les voiles. La voile. "Après une navigation de trois mois, ils arrivaient. C'était un trajet dangereux. (...) Vous paierez 6000 livres dit l'ordre royal du 12 mars 1534 au pilote Jacques Cartier qui va aux Terres Neuves découvrir certaines îles et pays où l'on dit qu'il se doit trouver grande quantité d'or". (ROBIN, 1983, p. 22)

De fait, la narratrice dit de multiples façons son désir de partir, mais où et vers quoi? Impossible de le dire puisqu'elle passe d'un souvenir à un autre, d'une histoire qui lui est personnelle à d'autres qui sont, elles, collectives. Ce désir de s'en aller la mène de Paris à Montréal, de Montréal à Paris, inter-changeant sans cesse la destination et le lieu de départ. En faisant allusion à la grande Histoire, à l'occupation nazie en France et à la découverte du Canada, la narratrice passe d'un souvenir - ici, l'occupation nazie - qui la constitue comme sujet immigrant à un souvenir associé à une histoire qui lui restera toujours étrangère - celle de la fondation du Canada. Il y a un brouillage mémoriel qui s'engendre dans ce passage d'une remémoration à une autre, lorsque passé et présent se confondent, entraînant dans ce mouvement déstabilisant, quelque chose comme le ratage de l'expérience de l'exil. Car, celle qui n'est pas à même de se souvenir d'où elle vient et où elle va est certainement en exil, mais un exil sans référent, précaire, qui correspondrait à l'impossibilité de l'unité (même fragile) du sujet immigrant à jamais

\footnotetext{
9 Simon Harel, "Montréal, une 'parole' abandonnée. Gérard Étienne et Régine Robin", p. 164.
} 
morcelé. D'ailleurs, au niveau du récit, l'unité devient inconcevable, comme l'éclatement de la ville et la langue multiple l'ont révélé. Simon Harel souligne avec pertinence: "La structure du roman témoigne d'un reconquête identitaire impossible" 10 . Il en va de même pour la remémoration qui, plutôt que d'aider la femme immigrante à se rappeler qui elle est, ne cesse de la défaire, de la fragmenter. L'identité est morcelée par la mémoire.

L'écriture de La Québécoite contribue nettement à accentuer l'impression de dissonance. Régine Robin pratique le collage, elle juxtapose les lieux et passe de l'un à l'autre sans lien logique, suivant des associations d'idées, des bribes de souvenirs qui l'assaillent ou les préoccupations d'un cours à préparer sur les auteurs juifs soviétiques. Paris est souvent en toile de fond, les stations de métro du quartier Latin continuent à "lui dire quelque chose", il est vrai que la nomination des lieux est liée à l'histoire / Histoire, certaines évoquent le théâtre des évènements de 1968, d'autres comme la station Grenelle déclenchent le souvenir terrible de la rafle des juifs au Vel'd'hiv, le 15 juillet 1942, et s'impose à sa mémoire. Parfois, au détour d'une phrase, remonte en elle comme une bouffée, le shetl de Varsovie où elle n'a pourtant jamais vécu mais que sa mère et sa tante lui ont raconté; de même, au cours de ses pérégrinations, tandis qu'elle prépare mentalement ses cours, réaffleure à son esprit la mémoire juive collective, la langue yiddish que lui parlait sa mère, les persécutions raciales, toute une mémoire étouffée, refoulée que l'écriture révèle. La couverture du roman - un choix de l'éditeur, semble-t-il - est un paysage naïf d'Europe centrale de Chagall, reproduit en page de garde et à la fin du livre. Ce tableau réitéré trois fois enveloppe significativement le texte et est l'emblème d'un passé - la Pologne - qui est celui de celle qui écrit, bref un télescopage continuel, voire l'impossibilité de faire le travail du deuil du pays d'origine:

\begin{abstract}
Désormais le temps de l'ailleurs de l'entre trois langues, trois alphabets dans la même journée. Télescopage de passages des grandes plaines de Russie, aux toits de Paris, de l'east end de Londres au lower east side new-yorkais, la Vistule, la Volga, la Volgule, la virgule, coma. L'oubli, l'amnésie. Collages. Tout se chevauche et se mêle. Désormais le temps de la confusion, de la contradiction, du désespoir solitaire. (ROBIN, 1983, p. 129)
\end{abstract}

Par une écriture dysphorique, volontairement très désarçonnante, fondée essentiellement sur l'accumulation et les jeux de mots - on a parfois l'impression d'un

\footnotetext{
$\overline{10}$ Simon Harel, "La parole orpheline de l'écrivain migrant", p. 409.
}

exercice de style qui déconstruit le parcours du texte (POTVIN, 2007) et qui lui donne un rythme haché, rendant bien le malaise culturel et symbolique de la jeune narratrice - La Québécoite nous conduit à réfléchir aux sens et aux aléas de l'immigration choisie.

\section{Conclusion}

Dans La Québécoite, l'incipit devient un leitmotiv unificateur: il ne rend pas compte seulement de l'espace urbain et de la structure du récit, mais également de la mémoire désarticulée: "Pas de lieu, pas d'ordre/Mémoire divisée à la jointure des mots/Les couches muettes du langage, brisées / La parole immigrante en suspens / entre / deux HISTOIRES" (ROBIN, 1983, p. 152). La rupture de la linéarité est évidente. Une fois divisée, la mémoire ne peut plus être unifiée. De cette manière, elle fait surgir l'entre-deux mémoriel de la grande Histoire, du passé dont la narratrice ne parvient pas à se détacher dans le présent. Le passé européen persiste à faire irruption dans l'espace du Nouveau Monde, des Amériques, comme pour rappeler la rupture, mais également l'horreur, l'impossible:
Il n'y a pas de métaphore pour signifier Auschwitz pas de genre, pas d'écriture. Écrire postule quelque part une cohérence, une continuité, un plein du sens [...]. Rien qui puisse dire l'horreur et l'impossibilité de vivre après. Le lieu entre le langage et l'Histoire s'est rompu. Les mots manquent. Le langage n'a plus d'origine ni de direction. Quel temps employer? Il n'y a qu'un présent éternel. Un présent qui ne passe pas. Le poids de ces millions de morts m'étouffe. En errance d'Europe en Amérique avec ces morts encombrants qui réclament leur dû dans un silence assourdissant. (ROBIN, 1983, p. 141)

Finalement, la mémoire d'un passé obsédant parce qu'impossible à oublier - force le déplacement, l'errance qu'elle soit spatiale ou langagière. Aux prises avec l'errance, la narratrice et le personnage trouvent dans l'exil un espace qui ne peut pas être véritablement nommé, mais qui est un espace tout de même: celui de l'entre-deux. Femme étrangère, autre, en exil à Montréal, femme autre à Paris, la Québécoite - tout comme l'écrivaine Régine Robin - se définit par le mouvement entre cette ville-ci et cette ville-là, entre un récit et un autre, entre le passé et le présent. Elle erre dans l'entredeux ouvert sur le multiple; elle emprunte des voies sinueuses, pas de chemin en ligne droite. D'ailleurs, la narratrice se déplace en oscillant, en s'approchant de ceci pour l'éloigner de cela, puis s'approchant de cela pour s'éloigner de ceci. Par ces itinéraires de vie et d'écriture, dans le mouvement du désordre intérieur et extérieur, 
une voix se fait entendre, voix incertaine et fragile, qui hésite et parfois perd son souffle. Mais cette voix résonne malgré tout, ébranle les certitudes, parvient à inscrire... l'expérience de l'immigrante, "les mots de l'autre-espace. Ils n'ont plus de place" (ROBIN, 1983, p. 86).

\section{Références}

ALLENDE, Isabel. Hija de la fortuna. Buenos Aires: Editorial Sudamericana, 1999.

ANDRADE, Oswald de. Anthropophagie. Paris: Flammarion, 1982 (1 ère èd. 1928).

BOTCHORICHVILI, Elena. Seulement attendre et regarder. Montréal: Boréal, 2012.

BROSSARD, Nicole et L. GIROUARD, (sous la dir. de). Anthologie de la poésie des femmes au Québec. Montréal: Les éditions du remue-ménage, 1992.

DERRIDA, Jacques. "Circonfessions", dans Jacques Derrida et Geoffrey Bennington (éds.) Jacques Derrida. Paris: Seuil, 1991. p. 26-52.

FRÉDÉRIC, Madeleine. “L'Écriture mutante dans $\mathrm{La}$ Québécoite de Régine Robin". Voix et Images, v. VXI, n. 3, p. 493-502, 1991.

HAREL, Simon. "La parole orpheline de l'écrivain migrant", dans Pierre Nepveu et Gilles Marcotte (éds.). Montréal imaginaire. Ville et littérature. Montréal: Fides, 1992. p. 373-418.

HAREL, Simon. 'Montréal, une 'parole' abandonnée. Gérard Étienne et Régine Robin", dans Benoît Melançon et Pierre Popovic (sous la dir. de), Montréal 1642-1992. Le Grand passage. Montréal: XYZ, 1994. p. 154-172.

HAREL, Simon. L'Étranger dans tous ses états. Enjeux culturels et littéraires. Montréal: XYZ, 1992.

L'HÉRAULT, Pierre, “Pour une cartographie de l'hétérogène: dérives identitaires des années 1960", dans Fictions de l'identitaire au Québec, sous la direction de Sherry SIMON et al. Montréal: XYZ, 1991. p. 43-64.
KRISTEVA, Julia. Étrangers à nous-mêmes. Paris: Fayard, 1988.

IMBERT, Patrick. Rencontres multiculturelles. Imprévus et coïncidences. Le Canada et les Amériques. Ottawa: Éditions de la Chaire de recherche de l'Université d'Ottawa: Canada: enjeux sociaux et culturels dans une société du savoir, 2013.

IMBERT, Patrick. "Le texte littéraire et la transculturalité", dans Le transculturel et les littératures des Amériques. Le Canada et les Amériques. Ottawa: Éditions de la Chaire de recherche de L'Université d'Ottawa: Canada: enjeux sociaux et culturels dans une société du savoir, 2012, p. 19-56.

IMBERT, Patrick. Comparer le Canada et les Amériques. Des racines aux réseaux transculturels. Québec: PUL, 2014.

POTVIN, Claudine. "La (dé)construction de la mémoire: $L a$ Québécoite de Régine Robin", dans L. LEQUIN, M.VERTHUY (sous la dir. de), Multi-culture, multi-écriture. La voix migrante au féminin en France et au Canada. Paris: L'Harmattan, 1996. p. 261-274.

ROBIN, Régine. La Québécoite. Montréal: Québec-Amérique, 1983.

ROBIN, Régine. Le Roman mémoriel: de l'histoire à l'écriture du hors lieu. Montréal: Le Préambule, 1989.

ROBIN, Régine. Le deuil de l'origine. Une langue en trop, une langue en moins. Paris: Presses Universitaires de Vincennes, 1993.

ROBIN, Régine. Nous autres, les autres. Montréal: Boréal, 2013.

SIMON, Sherry, Pierre L'HÉRAULT, Robert SCHWARTZWALD et Alexis NOUSS (sous la dir. de). Fictions de l'identitaire au Québec. Montréal: XYZ, 1991.

TASSINARI, Lamberto. "Sens de la transculture", dans Le projet transculturel de 'Vice Versa', sous la direction de A.P. MOSSETTO, séminaire du CISQ (2005). Bologna: Pendragon, 2006. p. 17-30.

Recebido: 25 de abril de 2015

Aprovado: 15 de julho de 2015

Contato: a.balint-babos@uwinnipeg.ca 